\title{
Increased p21 expression in chondrocytes of achondroplasic children independently from the presence of the G380R FGFR3 mutation
}

\author{
Antonina Parafioriti ${ }^{1}$, Silvia Del Bianco ${ }^{1}$, Donatella Barisani², Elisabetta Armiraglio ${ }^{1}$, \\ Giovanni Peretti ${ }^{3}$, and Walter Albisetti ${ }^{3}$ \\ ${ }^{1}$ Pathology Department, Orthopaedic Institute Gaetano Pini, piazza Cardinal Ferrari 1, 20122 Milan, Italy \\ ${ }^{2}$ Department of Experimental Medicine, Faculty of Medicine, University of Milano Bicocca, via Cadore 48, 20052 Monza (MI), Italy \\ ${ }^{3}$ Orthopaedic Division, Faculty of Medicine, University of Milano and Orthopaedic Institute Gaetano Pini, Milan, Italy
}

\begin{abstract}
Background. Achondroplasia $(\mathrm{ACH})$ represents the major cause of dwarfism and is due to mutations in the fibroblast growth factor receptor 3 (FGFR3) gene. The cellular mechanisms involved in the reduced growth have been mainly described for in vitro or in vivo models, but few data have been obtained for humans.

Methods. Thirteen children with ACH were enrolled in the study; the presence of FGFR3 mutations was determined by restriction fragment length polymorphism analysis and sequencing, whereas protein expression in cartilage biopsy was assessed by immunohistochemistry.

Results. Chondrocytes in cartilage biopsies of ACH children were characterized by the presence of growth arrest mediated by STAT activation (both STAT1 and STAT5) and increased expression of p21 and cyclin D1, whereas no expression of either p53 or cyclin D3 could be detected. This mechanism was present in $\mathrm{ACH}$ children carrying the G380R mutation but also in a patient in whom no mutation could be detected in the entire coding region of the FGFR3 gene.

Conclusions. These data thus demonstrate the presence of a common final mechanism involving p21 and possibly leading to a block in chondrocyte proliferation.
\end{abstract}

\section{Introduction}

Achondroplasia (ACH, MIM\# 100800), a common autosomal dominant skeletal dysplasia characterized by short stature, has a frequency of 1:26000 live births. ${ }^{1}$ More than $90 \%$ of the cases are sporadic, and there is a strong correlation with advanced paternal age, particularly over 35 years, suggesting that de novo mutations have mainly a paternal origin. ${ }^{2}$

Achondroplasia is part of a spectrum of disorders with variable clinical traits, including severe achondroplasia with developmental delay and acanthosis nigri-

Offprint requests to: $\mathrm{S}$. Del Bianco

Received: January 14, 2009 / Accepted: March 30, 2009 cans (SADDAN) and thanatophoric dysplasia (TD). ${ }^{3-5}$ These disorders are characterized, in most cases, by the presence of mutations in the coding region of fibroblast growth factor receptor 3 (FGFR3); in particular, a G380R transition within the transmembrane domain has been detected in most $\mathrm{ACH}$ patients, ${ }^{6}$ whereas mutations affecting codon 650 are able to cause severe skeletal dysplasias such as TDII (K650E) and SADDAN $(\mathrm{K} 650 \mathrm{M}){ }^{7}$

Fibroblast growth factor (FGF) receptors are tyrosine kinases (RTKs), which play a pivotal role in mediating growth, differentiation, and cell migration in various cell types. ${ }^{8}$ Binding of an extracellular ligand FGF leads to receptor dimerization and subsequent tyrosine phosphorylation, ${ }^{9}$ followed by activation of various signal transduction proteins and pathways, such as STAT1, STAT3, STAT5, ERK1, ERK2, MAPK, phospholipase C, protein kinase C, Src, phosphatidylinositol 3-kinase, and Akt. ${ }^{10-14}$

FGF signaling is one of the main pathways that control bone development, ${ }^{8}$ in particular bone size, as supported by the findings observed in FGFR3 knockout (KO) mice, in which severe bone dysplasia with enhanced growth due to expansion of proliferating chondrocytes in the growth plate is detected, ${ }^{15,16}$ Activation of FGFR3 inhibits chondrocyte proliferation and differentiation, as was also reported in studies on mouse models ${ }^{17,18}$; this growth inhibition is mediated by phosphorylation of STAT1, which then translocates in the nucleus and activates the cell cycle inhibitor p21Waf1/ Cip1. ${ }^{10-12,19}$ FGFs can also control bone growth through activation of the MAPK-ERK1/2 pathway in chondrocytes, which affects longitudinal growth by regulating hypertrophic chondrocyte differentiation and matrix deposition. ${ }^{20}$ FGFR3 mutations, such as those detected in achondroplasia and in TD have been shown to trigger STAT(s) signaling, increase expression of p21 in mouse and human cartilage models, ${ }^{10,11}$ and activate the MAP kinase pathway. ${ }^{10}$ However, up to now, most of the 
studies have been performed on mouse or in vitro culture models, with only one article on analyzing this pathway in human fetuses. ${ }^{11}$ Thus, validation of these findings in the cartilage of children with achondroplasia is still lacking.

The aim of this study was to characterize the genetic defect and evaluate the morphology and expression of proteins involved in the cell cycle in cartilage biopsies of achondroplasic children undergoing surgery for limb elongation.

\section{Methods}

\section{Patient selection}

Thirteen children (three males, ten females) with a clinical diagnosis of achondroplasia followed at the Istituto Ortopedico Gaetano Pini (Milan, Italy) were enrolled in the study. All patients were admitted to the hospital to undergo a limb elongation surgical procedure. As controls, we employed DNAs $(n=10)$ and biopsy specimens $(n=8)$ obtained from age-matched controls undergoing surgery for a malignant neoplasm. The surgical samples were obtained from patients with osteosarcoma affecting the long bones (tibia and femur) but not involving articular cartilage. All of these subjects underwent chemotherapy before surgery according to standard international protocols.

The patients and their families were informed that data from the study would be submitted for publication and gave their consent. The study protocol conforms to the ethical guidelines of the 1975 Declaration of Helsinki as reflected in a priori approval by the Ethics Committee of the Istituto Ortopedico Gaetano Pini.

\section{DNA extraction and detection of the G380R mutation}

Genomic DNA was extracted from peripheral blood leukocytes using the Wizard genomic DNA purification kit (Promega, Madison, WI, USA), according to the manufacturer's instructions. Initial screening for detection of the G308R mutation was performed using a modification of the method described by Lanning and Brown. ${ }^{21}$ Briefly, $200 \mathrm{ng}$ of DNA was used as a template for a polymerase chain reaction (PCR) performed using specific primers ${ }^{21}$ (final concentration $200 \mathrm{nM}$ ) in the presence of $1.5 \mathrm{mM} \mathrm{MgCl}_{2}, 200 \mathrm{mM}$ each dNTP, and 2 units of Taq polymerase (Red Taq; Sigma-Aldrich, Milan, Italy). Amplification was performed, after initial heating to $94^{\circ} \mathrm{C}$ for $5 \mathrm{~min}$, at $94^{\circ} \mathrm{C}$ for $2 \mathrm{~min}$, at $65^{\circ} \mathrm{C}$ for $1 \mathrm{~min}$, and at $72^{\circ} \mathrm{C}$ for 1 min over 40 cycles in a Perkin Elmer (system 2700) thermocycler, with a final extension of $7 \mathrm{~min}$ at $72^{\circ} \mathrm{C}$. PCR amplification products were then separated on a $2 \%$ agarose gel to verify the correct size of the product (132 bp) and digested with 10 units of BsrGI (New England Biolabs, Beverly, MA, USA) at $37^{\circ} \mathrm{C}$ overnight. Digestion products were separated on a $3 \%$ agarose gel, and in presence of the mutation we detected 112- and 20-bp fragments.

\section{Sequencing the FGFR3}

To detect mutations different from the G380R, we sequenced the entire coding region of the FGFR3 (exons 2-18). DNA regions containing exons and intron-exon junctions were amplified using the primers described by Wüchner et al., ${ }^{22}$ but in the case of exon 9 we also designed a new pair of primers (forward 5'-CCTCTCCTCGTCTCTGCTCA-3' and reverse 5'-CCTCTGACTGGTGGCTGTTT-3') for better evaluation of the intron-exon junctions. All additional primers were designed using the Oligo Explorer software. Amplifications were performed as described above, using different annealing temperatures according to the primer pairs (details available on request). PCR conditions for exon 9 using the newly designed primers were at $94^{\circ} \mathrm{C}$ for $5 \mathrm{~min}$, at $94^{\circ} \mathrm{C}$ for $1 \mathrm{~min}$, at $60^{\circ} \mathrm{C}$ for $1 \mathrm{~min}$, and at $72^{\circ} \mathrm{C}$ for 2 min over 35 cycles. Amplified products were purified using ion-exchange columns (Qiaquick; Qiagen, Milan, Italy), and sequencing was performed using the ABI Prism terminator cycle sequencing ready reaction kit on an ABI Prism 3100 Avant DNA sequencer (PE; Applied Biosystems, Foster City, CA, USA). All exons were sequenced using forward and reverse primers; the obtained sequences were aligned and independently manually checked by two of the authors (S.D.B., D.B.). Exons 7, 9, and 10 were sequenced twice, using as the template two different blood samples obtained from the patient not carrying the G380K mutation. Every sequence was compared with its corresponding reference sequence available in GeneBank (NM_000142.2 for FGFR3).

\section{Biopsy processing and evaluation}

Biopsy specimens were obtained from articular cartilage, fixed in a $10 \%$ solution of paraformaldehyde in

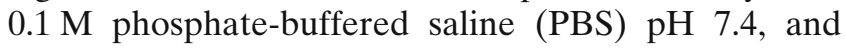
embedded in paraffin. Sections were mounted on polylysine- or silane-treated slides (Sigma, Milan, Italy) for hematoxylin and eosin (H\&E) staining and immunohistochemistry. The sites of the biopsies are reported in Table 1 . For ethical reasons, only small biopsy specimens could be obtained during surgery; thus, we could not perform a classic histomorphometric analysis with computerized evaluation of the images. To quantify the number of chondrocytes present in controls and $\mathrm{ACH}$ patients, the cells present in four independent $400 \times$ fields were counted for each specimen, and an average 


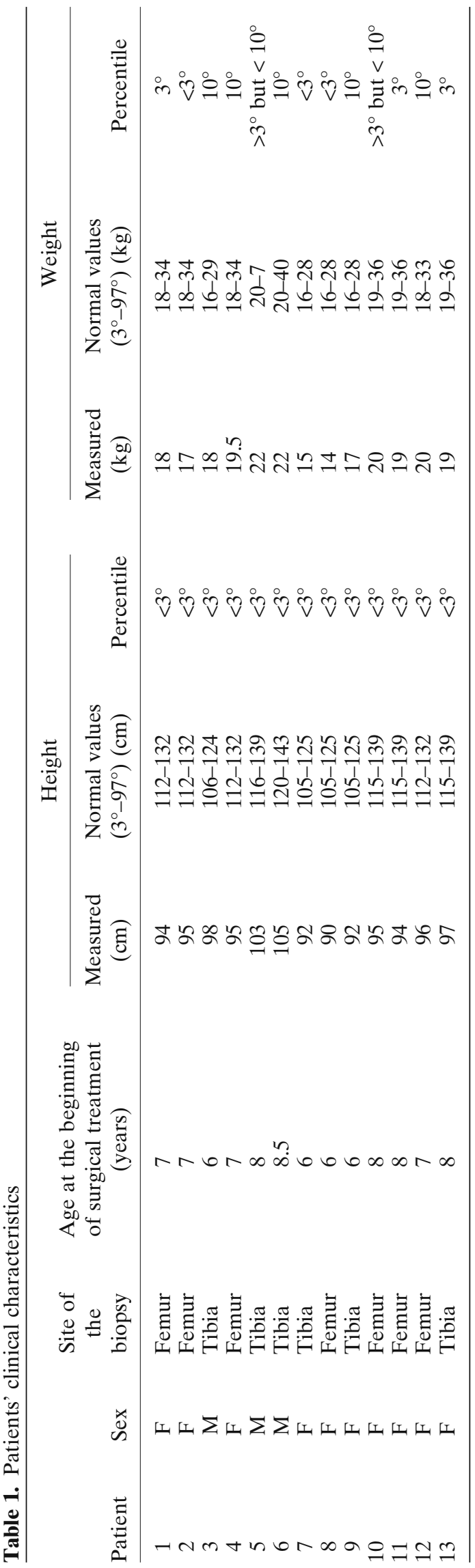

for each subject was calculated. Possible differences between the groups was evaluated by Student's $t$-test using SAS System Software (version 9.1) (SAS Institute, Cary, NC, USA).

\section{Immunohistochemistry}

Immunohistochemistry was performed on all the subjects enrolled in the study. Briefly, slides were pretreated with $3 \% \mathrm{H}_{2} \mathrm{O}_{2}$ for 15 min to inhibit endogenous peroxidase and with a blocking agent (Normal Reagent; Vector, Burlingame, CA, USA) for $30 \mathrm{~min}$ to prevent background staining. Unmasking of the antigens was performed by incubating tissue sections in $10 \mathrm{mM}$ sodium citrate buffer $\mathrm{pH} 6$ for $9 \mathrm{~min}$. Incubation with primary antibodies was performed for $1 \mathrm{~h}$ at room temperature using the following antibodies: anti-S100 (1:200; Dako, Glostrup, Denmark), anti-STAT1 (1:200, Cell Signaling, Danvers, MA, USA), anti-STAT5 (1:200, Cell Signaling), anti-p21 (1:100, Cell Signaling), anti-p53 (1:125; Dako), anti-cyclin D1 (1:200, Cell Signaling), and anti-cyclin D3 (1:100, Cell Signaling). Detection of the hybridization signal was performed with biotinylated secondary antibodies [anti-rabbit immunoglobulin (IgG) or anti-mouse $\mathrm{IgG}$ ] (Vector Elite Kit; Vector Laboratories, Burlingame, CA, USA), followed by peroxidase-conjugated streptavidin and diaminobenzidine solution (ABC detection system, Vector). Counterstaining was performed with Harris hematoxylin. Sections were examined independently by two pathologists (A P and EA) unaware of patients' genotype.

\section{Results}

\section{Patients' clinical and radiological characteristics}

The clinical features of the 13 achondroplasic children are reported in Table 1. All patients showed a decreased bone growth, with a stature below the 3rd percentile, with a weight that was below the 10th percentile. As reported in Table 2, all patients showed clinical signs typical of achondroplasia, such as macrocephaly, rhizomelic shortening of all the limbs, frontal bossing, midface hypoplasia, and nasal bridge depression. Lower limb deformity was present in most patients, whereas other manifestations, such as trident hand, were rarer. As also described in Table 2, radiological signs of achondroplasia were detected in various percentages in all patients.

\section{Genetic analysis of the FGFR3}

We performed an initial screening to identify the presence of the G380R mutation in $\mathrm{ACH}$ patients using 
Table 2. Patients' clinical and radiological signs

\begin{tabular}{|c|c|c|c|c|c|c|c|c|c|c|c|c|c|}
\hline Signs & 1 & 2 & 3 & 4 & 5 & 6 & 7 & 8 & 9 & 10 & 11 & 12 & 13 \\
\hline \multicolumn{14}{|l|}{ Clinical signs } \\
\hline Macrocephaly & + & + & + & + & + & + & + & + & + & + & + & + & + \\
\hline Frontal bossing & + & + & + & + & + & + & + & + & + & + & + & + & + \\
\hline Midface hypoplasia & + & + & + & + & + & + & + & + & + & + & + & + & + \\
\hline Nasal bridge depression & + & + & + & + & + & + & + & + & + & + & + & + & + \\
\hline Rhizomelic shortening of all limbs & + & + & + & + & + & + & + & + & + & + & + & + & + \\
\hline "Trident" hand & + & + & + & - & + & + & - & - & + & - & - & - & - \\
\hline Elbow contracting during bending & + & + & + & + & + & + & - & + & + & + & + & + & + \\
\hline Thoracolumbar gibbus & + & + & + & - & + & + & + & + & - & - & - & + & + \\
\hline Vertebral canal stenosis & - & - & - & - & - & - & - & - & - & - & - & - & - \\
\hline Lower limbs deformity & + & - & + & + & + & + & - & + & + & + & + & + & + \\
\hline \multicolumn{14}{|l|}{ Radiological signs } \\
\hline Interpedicular distance decrease & + & + & - & + & + & + & + & + & + & + & + & + & - \\
\hline Pelvic diameter increase & + & + & + & + & + & + & + & + & + & - & - & - & + \\
\hline Calf bone longer than shinbone & - & - & + & + & - & + & + & + & + & - & - & + & + \\
\hline Rhizomelic shortening of all the limbs & + & + & + & + & + & + & + & + & + & + & + & + & + \\
\hline Vertebral column deformity & + & + & + & - & + & + & + & + & - & + & + & + & + \\
\hline Lower limb deformity & + & - & + & + & + & + & - & + & + & + & + & + & + \\
\hline
\end{tabular}

restriction enzyme digestion of the amplified DNA region harboring the mutation. Among the 13 analyzed patients, 12 carried the G380R mutation in heterozygosis, as demonstrated by the presence of the uncut (wildtype) and cut (mutated) alleles on gel electrophoresis (data not shown). In one patient 13, the mutation was not detected; we first sequenced the amplified product to confirm the lack of mutation and then performed an analysis of the entire coding region to assess whether other mutations were present in the exons codifying for the extracellular, transmembrane, or cytoplasmic region of the FGFR3 protein. Although exons 2-18 and intron/ exon junctions were sequenced in both forward and reverse, we could not detect any mutation.

\section{Morphological evaluation of cartilage biopsies}

Cartilage biopsy specimens obtained during surgery were first analyzed using standard histology techniques (Fig. 1). Compared to cartilage obtained from agematched controls with normal growth, the samples from achondroplasic patients showed a reduced number of chondrocytes, with shortening of normal column placement and of transitional calcification areas (Fig. 1C,D). The average number of chondrocytes, as determined in a $400 \times$ field, was $21.75 \pm 2.55$ in control samples compared to $16.35 \pm 2.34$ in $\mathrm{ACH}$ biopsies $(P<0.05)$. A similar pattern was observed even in the subject who was negative for mutations in the FGFR3 gene (data not shown).

S-100 expression was assessed by immunohistochemistry, and similar levels of expression were observed in chondrocytes in control subjects and G380R mutated patients (data not shown). No significant difference in
S-100 expression was detected in the only patient with wild-type FGFR3 sequence when compared to controls or other achondroplasic patients (data not shown).

\section{Immunohistochemical evaluation of cell cycle proteins}

To assess whether the presence of a G380R was associated with an activation of the FGFR3 pathway. we evaluated the expression of STAT1, a transcription factor that has been identified as one of the main regulators of bone growth. As shown in Fig. 2A, STAT1 expression was barely detectable in chondrocytes of controls subjects, whereas its expression was strongly augmented in biopsies of $\mathrm{ACH}$ patients, with increased staining in the nuclear region, compatible with nuclear translocation of the transcription factor (Fig. 2B). Interestingly, even in the absence of FGFR3 mutations, a signal for STAT1 could be detected, although possibly not as strong as that detected in G380R patients (Fig. 2C). We also evaluated STAT5 expression, which was almost absent in controls; it was increased in patients with the G380R mutation (Fig. 3A,B). Unfortunately, in the patient with no FGFR3 mutations, immunohistochemistry using the anti-STAT5 antibody did not give consistent results (data not shown). To block cell cycle progression, STAT1 acts on p21; thus, we evaluated p21 expression, which was not detectable in control biopsies (Fig. 4A) but was strongly expressed in chondrocytes of achondroplasic patients independently from the presence or absence of the G380R mutation (Fig. 4B,C). The p 21 expression can also be regulated by p53 and for this reason we evaluated, by immunohistochemistry, whether p53 protein was up-regulated in subjects with achondroplasia. This analysis did not reveal any 


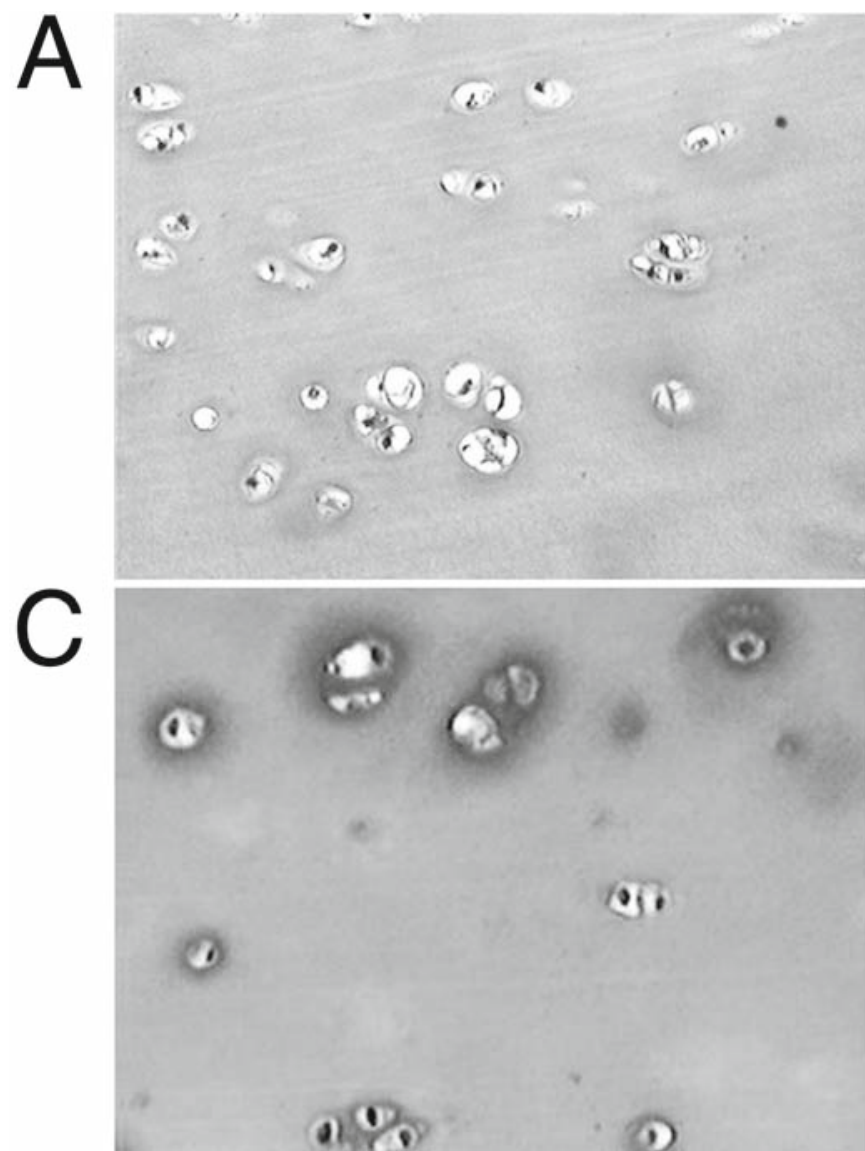

Fig. 1. Morphological evaluation of cartilage biopsy specimens. A Control epiphyseal cartilage $(\times 400)$. B Control transition cartilage $(\times 200)$. C Achondroplasic epiphyseal cartilage
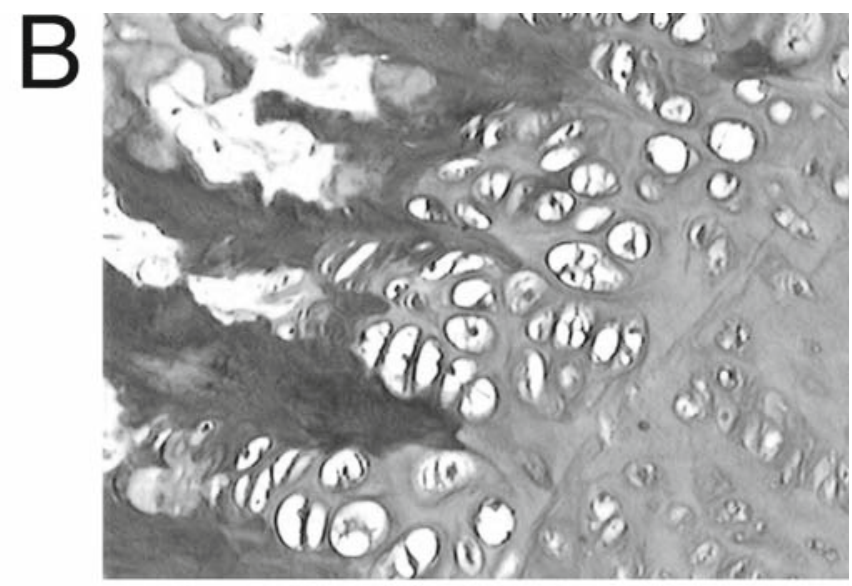

D

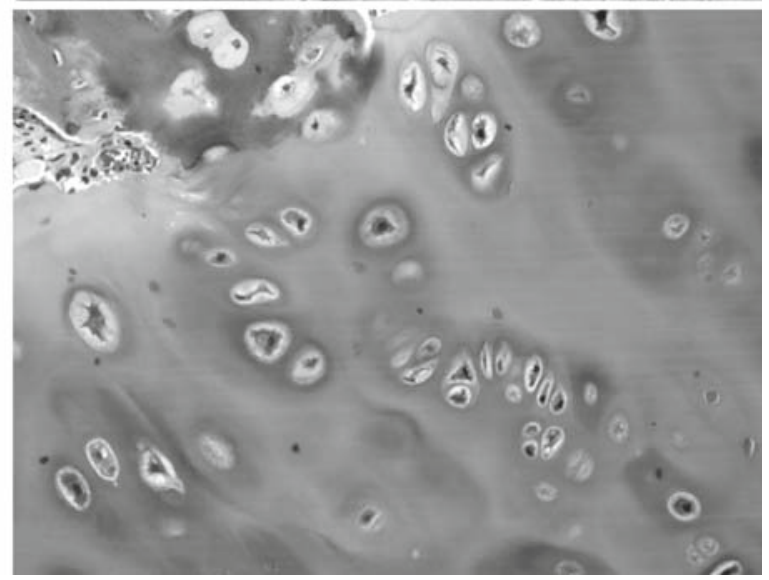

(patient with G380R mutation) $(\times 400)$. D Achondroplasic transition cartilage (patient with G380R mutation) showing a reduced number of chondrocytes $(\times 200)$
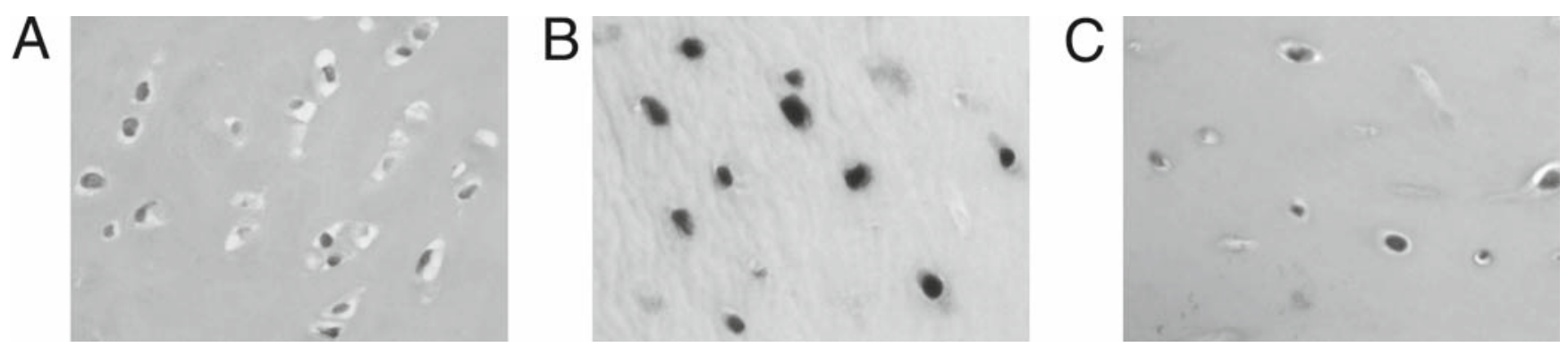

Fig. 2. Immunohistochemistry analysis of STAT1 expression. A Control epiphyseal cartilage with no signal. B Achondroplasic epiphyseal cartilage (patient with G380R mutation)

significant difference between controls and $\mathrm{ACH}$ patients, showing a very low level (almost undetectable) of expression of p53 (data not shown). Up-regulation of p21 suggests arrest of cell cycle progression; thus, we analyzed the expression of two cyclin $\mathrm{D}$ subtypes, which are essential in the $\mathrm{G}_{1}-\mathrm{S}$ phase transition. Cyclin D1 expression was not detectable in control biopsies, shows increased STAT1 expression. C Achondroplasic epiphyseal cartilage (patient without G380R mutation). (A-C $\times 400)$ whereas intense positive staining was present in all ACH patients, with or without FGFR3 mutations (Fig. 5).

As regards cyclin D3, no immunostaining signal could be identified either in controls or in $\mathrm{ACH}$ patients, suggesting the lack of accumulation of this protein in the analyzed chondrocytes (data not shown). 

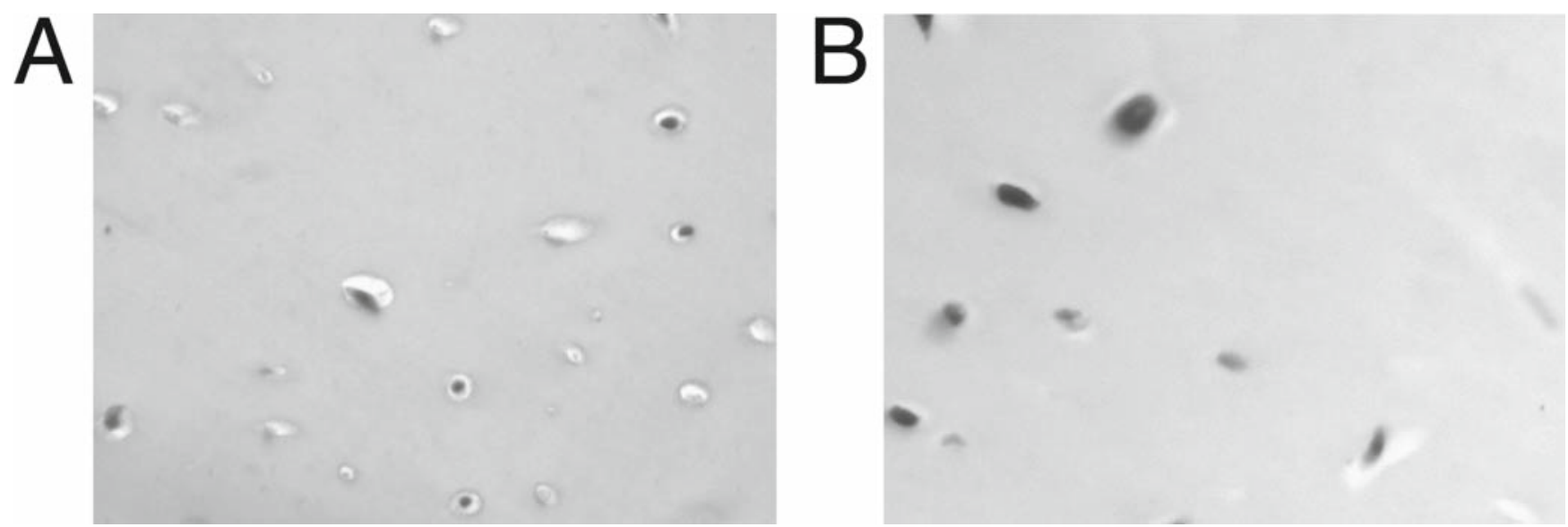

Fig. 3. Immunohistochemistry analysis of STAT5 expression. A Control epiphyseal cartilage with no detectable STAT5 expression. B Achondroplasic epiphyseal cartilage (patient with G380R mutation) shows increased STAT5 expression. (A, B $\times 400)$
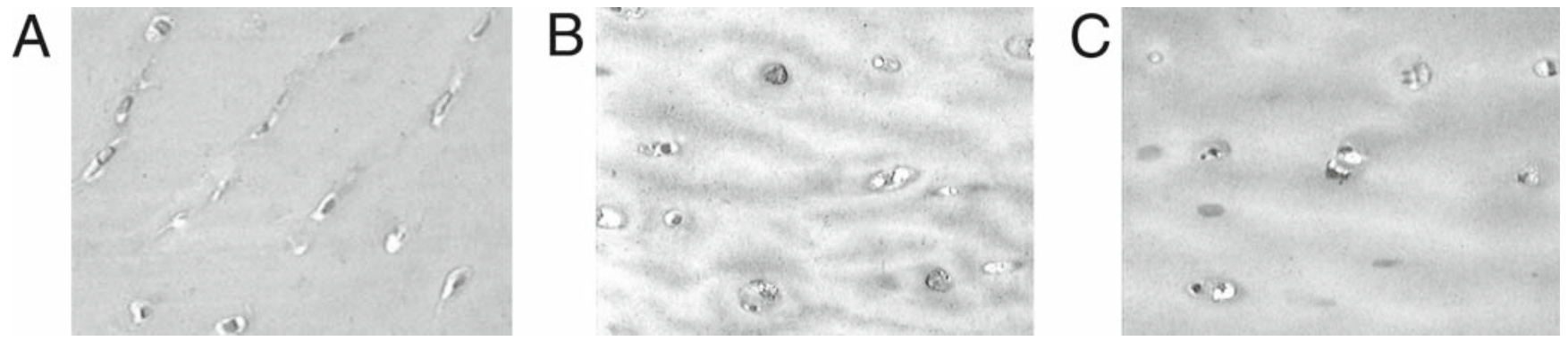

Fig. 4. Immunohistochemistry evaluation of p21 expression. A Control epiphyseal cartilage with no signal. B Achondroplasic epiphyseal cartilage (patient with G380R mutation) shows increased p21 expression. C Achondroplasic epiphyseal cartilage (patient without G380R mutation) with increased p21 expression. $(\mathbf{A}-\mathbf{C} \times 400)$

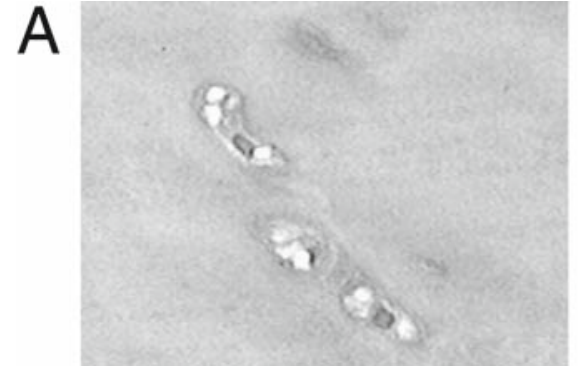

Fig. 5. Immunohistochemistry analysis of cyclin D1 expression. A Control epiphyseal cartilage with no signal. B Achondroplasic epiphyseal cartilage (patient with G380R mutation).

\section{Discussion}

Achondroplasia represents one of the most common forms of dwarfism and is generally due to mutations in the FGFR3 gene. Several studies performed with in vitro and in vivo models have provided, during the last few years, insights regarding the pathogenetic mechanisms responsible for premature bone growth arrest. However, few studies have been performed on human samples. Here we describe the morphological and expression analysis of cartilage samples obtained from achondroplasic children, showing activation of the STAT pathway and detecting similar findings in patients with the G380R mutation and in one subject with no mutation detected in the entire coding region.

Achondroplasia is due, in most of the patients, to a point mutation causing an amino acid change (G380R) in the transmembrane domain of FGFR3, resulting in a 
gain of function and consequent activation of the downstream signaling cascade involving either STAT1 or the MAPK-ERK pathway. ${ }^{10-12}$ Among the 13 children analyzed here, 12 were carriers of the G380R mutation; the remaining child did not show any mutation in the FGFR3 gene, although the clinical characteristics and the clinical and radiological signs were identical to those observed in the other 12 patients. The sequencing of the entire coding region of the FGFR3 gene did not reveal the presence of mutations in the examined exons. The G380R mutation is detected in about $98 \%$ of $\mathrm{ACH}$ patients, although other, rarer mutations have been reported, such as Gly375Cys. ${ }^{23}$ A more recent article, Heuertz et $\mathrm{al}^{24}$ reported a patient with $\mathrm{ACH}$ who carried a single base substitution converting serine 279 into cysteine (S279C) in the third Ig loop of the extracellular portion of the receptor another mutation in the same proteic region was associated with a more severe phenotype in hypochondroplasia. This mutation, reported also by Friez and Wilson, ${ }^{25}$ is localized in exon 7. Both studies suggested that sequencing of this exon be included in the screening for $\mathrm{ACH}$ and $\mathrm{HCH}$. Another study on 24 Portuguese patients with $\mathrm{ACH}$ first sequenced exons 9 and 10, identifying the G380R mutation in 21 cases, a N540K substitution in two, and no mutation after sequencing of the entire gene in one subject. ${ }^{26}$ Although no mutation was detected in our patient, we could not exclude the possibility that variations at the transcriptional or posttranscriptional level could alter FGFR3 expression in the affected tissue (i. e., the cartilage); however, the amount of tissue obtained prevented us from performing these analyses.

STAT1 immunohistochemistry evaluation revealed the presence of the activated transcription factor in the cytoplasm as well as the nucleus of chondrocytes of patients with reduced bone growth, and in this case, independently from the presence of the G380R mutation. These findings are in agreement with those reported by Legeai-Mallet et al., who identified the same alteration in fetuses affected by $\mathrm{ACH}$ or TD. ${ }^{11}$ Hyperactivation of STAT1 thus remains through different ages, showing that FGFR3 pathway activation is present not only in fetuses but in young children. STAT1 activation is commonly regarded as able to block cell cycle progression through the induction of $\mathrm{p} 21,{ }^{12,16}$ although there may be some exceptions, as reported by Nowroozi et al. ${ }^{27}$ who, in a cellular model of thanatophoric dysplasia, showed that siRNA-induced STAT1 down-regulation was unable to affect $\mathrm{p} 21$ expression. In all of the examined ACH biopsies in our study, p21 expression was strongly increased compared to that of the control samples; interestingly, p21 hyperexpression was also detected in the patient with no FGFR3 mutation, suggesting that the mechanism leading to cessation of cell division uses a common final pathway. Increased expression of p21 has been previously reported in animal and cellular models of achondroplasia. ${ }^{10,12}$ Our findings in humans agree with those observed in the cartilage of fetuses affected by ACH or TD.${ }^{11}$ However, p21 could block the cell cycle as a response to different stimuli, and its expression could be also regulated by $\mathrm{p} 53$, which is activated in the presence of cellular damage (mostly DNA damage) and induces p21 transcription. ${ }^{28}$ We could not detect any increased expression or nuclear transmigration of p53 in the chondrocytes of the analyzed ACH patients, which supports the hypothesis that p21 hyperexpression is due to a primary alteration causing $\mathrm{ACH}$.

The effect of p21 on the cell cycle is mediated by a block of the $\mathrm{D}$ cyclin-CDK4 complex, which is essential for progression from the $\mathrm{G}_{1}$ to the $\mathrm{S}$ phase of growth. ${ }^{28}$ Although this block is usually associated with a reduction in $\mathrm{D}$ cyclin protein levels, ${ }^{29,30}$ it has also been reported that $\mathrm{p} 21$ can bind to the $\mathrm{D}$ cyclin-CDK4 complex and prevent it from functioning without inducing any significant alteration in the protein level of the components of the complex. ${ }^{28} \mathrm{We}$ observed increased expression of cyclin D1 in the ACH cartilage biopsies analyzed in this study, whereas cyclin D3 was not detectable. The cyclin D1 findings are thus in agreement with the possibility that p21 stabilizes the cyclin-CDK complex rather than inducing its degradation.

\section{Conclusion}

This study provides evidence regarding activation of the STAT pathway even in biopsies of achondroplasic children and that $\mathrm{p} 21$ hyperexpression may represent the final effector mechanism causing cell cycle arrest even in the absence of detectable FGFR3 mutation.

\section{References}

1. Stoll C, Dott B, Roth MP, Alembik Y. Birth prevalence rates of skeletal dysplasias. Clin Genet 1989;35:88-92.

2. Wilkin DJ, Szabo JK, Cameron R, Henderson S, Bellus GA, Mack ML, et al. Mutations in fibroblast growth-factor receptor 3 in sporadic cases of achondroplasia occur exclusively on the paternally derived chromosome. Am J Hum Genet 1998;63: $711-6$.

3. Tavormina PL, Bellus GA, Webster MK, Bamshad MJ, Fraley AE, McIntosh I, et al. A novel skeletal dysplasia with developmental delay and acanthosis nigricans is caused by a Lys650Met mutation in the fibroblast growth factor receptor 3 gene. Am J Hum Genet 1999;64:722-31.

4. Horton WA, Lunstrum GP. Fibroblast growth factor receptor 3 mutations in achondroplasia and related forms of dwarfism. Rev Endocr Metab Disord 2002;3:381-5.

5. Spranger JW, Brill PW, Poznanski A. Bone dysplasias: an atlas of genetic disorders of skeletal development. 2nd edn. New York: Oxford University Press; 2002. 
6. Bellus GA, Hefferon TW, Ortiz de Luna RI, Hecht JT, Horton WA, Machado M, er al. Achondroplasia is defined by recurrent G380R mutations of FGFR3. Am J Hum Genet 1995;56:368-73.

7. Tavormina PL, Shiang R, Thompson LM, Zhu YZ, Wilkin DJ, Lachman RS, et al. Thanatophoric dysplasia (types I and II) caused by distinct mutations in fibroblast growth factor receptor 3. Nat Genet 1995;9:321-8.

8. Ornitz DM, Marie JP. FGF signaling pathways in endochondral and intramembranous bone development and human genetic disease. Genes Dev 2002;16:1446-5.

9. Schlessinger J. Cell signalling by receptor tyrosine kinases. Cell 2000;103:211-25.

10. Su WC, Kitagawa M, Xue N, Xie B, Garofalo S, Cho J, et al. Activation of Stat1 by mutant fibroblast growth-factor receptor in thanatophoric dysplasia type II dwarfism. Nature 1997;386: 288-92.

11. Legeai-Mallet L, Benoist-Lasselin C, Munnich A, Bonaventure J. Overexpression of FGFR3, Stat1, Stat5 and p21Cip1 correlates with phenotypic severity and defective chondrocyte differentiation in FGFR3-related chondrodysplasias. Bone 2004;34:26-36.

12. Sahni M, Ambrosetti DC, Mansukhani A, Gertner R, Levy D, Basilico C. FGF signaling inhibits chondrocyte proliferation and regulates bone development through the STAT-1 pathway. Genes Dev 1999;13:1361-6.

13. Kong M, Wang CS, Donoghue DJ. Interaction of fibroblast growth factor receptor 3 and the adapter protein SH2-B. A role in STAT5 activation. J Biol Chem 2002;277:15962-70.

14. Shimoaka T, Ogasawara T, Yonamine A, Chikazu D, Kawano H, Nakamura K, et al. Regulation of osteoblast, chondrocyte, and osteoclast functions by fibroblast growth factor (FGF)-18 in comparison with FGF-2 and FGF- 10. J Biol Chem 2002;277: 7493-500.

15. Colvin JS, Bohne BA, Harding GW, McEwen DG, Ornitz DM. Skeletal overgrowth and deafness in mice lacking fibroblast growth factor receptor 3. Nat Genet 1996;12:390-7.

16. Deng C, Wynshaw-Boris A, Zhou F, Kuo A, Leder P. Fibroblast growth factor receptor 3 is a negative regulator of bone growth. Cell 1996;84:911-21.

17. Chen L, Adar R, Yang X, Monsonego EO, Li C, Hauschka PV, et al. Gly369Cys mutation in mouse FGFR3 causes achondroplasia by affecting both chondrogenesis and osteogenesis. J Clin Invest 1999;104:1517-25.

18. Wang Y, Spatz MK, Kannan K, Hayk H, Avivi A, Gorivodsky M, et al. A mouse model for achondroplasia produced by targeting fibroblast growth factor receptor 3. Proc Natl Acad Sci U S A 1999;96:4455-60.
19. Li C, Chen L, Iwata T, Kitagawa M, Fu XY, Deng CX. A Lys644Glu substitution in fibroblast growth factor receptor 3 (FGFR3) causes dwarfism in mice by activation of STATs and ink4 cell cycle inhibitors. Hum Mol Genet 1999;8:35-44.

20. Murakami S, Balmes G, McKinney S, Zhang Z, Givol D, de Crombrugghe B. Constitutive activation of MEK1 in chondrocytes causes Stat1-independent achondroplasia-like dwarfism and rescues the FGFR3-deficient mouse phenotype. Genes Dev 2004;18:290-305.

21. Lanning RW, Brown CA. An improved methodology for the detection of the common mutation in the FGFR3 gene responsible for achondroplasia. Hum Mutat 1997;10:496-9.

22. Wüchner C, Hilbert K, Zabel B, Winterpacht A. Human fibroblast growth factor receptor 3 gene (FGFR3): genomic sequence and primer set information for gene analysis. Hum Genet 1997; 100:215-9.

23. Horton WA, Hall JG, Hecht JT. Achondroplasia. Lancet 2007; 370:162-72.

24. Heuertz S, Le Merrer M, Zabel B, Wright M, Legeai-Mallet L, Cormier-Daire V, et al. Novel FGFR3 mutations creating cysteine residues in the extracellular domain of the receptor cause achondroplasia or severe forms of hypochondroplasia. Eur J Hum Genet 2006;14:1240-7.

25. Friez MJ, Wilson JA. Novel FGFR3 mutations in exon 7 and implications for expanded screening of achondroplasia and hypochondroplasia: a response to Heuertz et al. Eur J Hum Genet 2008;16:277-8.

26. Almeida MR, Campos-Xavier AB, Medeira A, Cordeiro I, Sousa AB, Lima M, et al. Clinical and molecular diagnosis of the skeletal dysplasias associated with mutations in the gene encoding fibroblast growth factor receptor 3 (FGFR3) in Portugal. Clin Genet 2009;75:150-6.

27. Nowroozi N, Raffion S, Wang T, Apostol BL, Bradshaw RA, Thompson LM. Sustained ERK1/2 but not STAT1 or 3 activation is required for thanatophoric dysplasia phenotypes in PC12 cells. Hum Mol Genet 2005;14:1529-38.

28. Olashaw N, Bagui TK, Pledger WJ. Cell cycle control: a complex issue. Cell Cycle 2004;3:263-4.

29. Kang H, Cui K, Zhao K. BRG1 controls the activity of the retinoblastoma protein via regulation of p21CIP1/WAF1/SDI. Mol Cell Biol 2004;24:1188-99.

30. Sugimoto M, Martin N, Wilks DP, Tamai K, Huot TJ, Pantoja C, et al. Activation of cyclin D1-kinase in murine fibroblasts lacking both p21(Cip1) and p27(Kip1). Oncogene 2002;21:8067-74. 